\title{
Análisis y representación del conocimiento: Aportaciones de la Psicología Cognitiva
}

\author{
Juan Antonio Bernad \\ Instituto de Ciencias de la Educación \\ Universidad de Zaragoza
}

\subsection{Resumen}

Esta ponencia se propone esclarecer algunas de las exigencias impuestas al documentalista a partir de modelos psicológicos que definen la actividad cognitiva de los sujetos en cuanto procesadores de información. El autor realiza un análisis del conocimiento humano desde cinco dimensiones: a) tipos de conocimiento utilizados por los humanos; b) influjo de la experiencia previa del sujeto; c) fases del pensamiento en la construcción de los productos que llamamos ciencia; d) estrategias de pensamiento utilizadas por los individuos; y e) influjo del factor afectivo o motivador en el proceso de pensar. Es impensable una transmisión eficaz de los conocimientos sin respetar esta "gramática mental" de los destinatarios. La poencia aboga por potenciar la investigación básica del conocimiento humano dando prioridad al papel de cada uno de los hemisferios en el procesamiento de información y a las etapas evolutivas de los sujetos en cuanto a sus posibilidades de pensar. (Autor)

Palabras clave: Ciencia Cognitiva. Organización del Conocimiento. Representación del Conocimiento. Psicología Cognitiva.

\subsection{Abstract}

The main aim of this lecture is to give light to some of the demands required to documentalists about the psychological models which define at present the cognitive activity of the human beings considered as processors of information. Taking into account the mentioned models frame, the author pays a special attention to the analysis of the human knowledge and its representation from five dimensions: a) the kinds of knowledge used by human beings; b) the influence of the individual's previous experience; c) the different stages of thought involved in the construction of the consolidated products that we call science; d) the strategies of thought used by human beings; and, at last, e) the influence of the motivational factor within the thinking process. It is unthinkable trying to transmit

Scire. $1: 1$ (en.-jun. 1995). 
thoughts without considering this set of requirements demanded by the cogntive activity of the users. This lecture encourages the basic research of human knowledge and its processes which tends to make clear the different levels of involvement of every hemisphere in the processing of information, the human beings' evolutionary stages referred to their prossibilities of thinking and their capacity of cognitive training and the cognitive links of interaction between the specific nature of the knowledge which must be assimilated. (Author)

Keywords: Cognitive Science. Knowlege Organisation. Knowledge Representation. Cognitive Psychology.

\section{Planteamiento inicial del tema: el binomio construcción-trasmi- sión del conocimiento científico}

Para enmarcar las reflexiones propuestas en este trabajo, nada mejor, quizás, que citar un par de hechos significativos que pueden ayudarnos a entender el binomio que encabeza este apartado introductorio.

Por los años 20, el joven Jean Piaget se trasladó desde su ciudad natal, Ginebra, a París y se puso a la órdenes de uno de los padres de los tests psicológicos, el Dr. Simon. Este encargó al ginebrino la traducción al francés de los tests del inglés Burt al objeto de aplicarlos a los niños parisinos y así contar con una base empírica que permitiera agruparlos en las aulas en función de sus capacidades intelectuales. En tales tests, aparecían items como éste" "Dijo el juez al reo, voy a colgarte para que te sirva de escarmiento".

El inteligente ayudante hizo puntualmente lo que le había encargado el Dr. Simon pero se preguntó, además, que ocurría en la mente de los niños para que unos respondieran al citado item fijándose solamente en la crueldad del juez y otros captaran la incongruencia del enunciado. Indagando durante más de medio siglo los procesos que median entre la recepción de estímulos por parte de los sujetos y las respuestas que dan a los mismos, Piaget pudo contestar a una pregunta que está en la entraña de nuestro tema: cómo se alcanza el conocimiento científico y cuáles son las leyes que lo explican. Nacía así la teoría genética de la inteligencia según la cual para asimilar cualquier conocimiento es imprescindible que los sujetos se comporten como seres activos y realicen determinados procesos internos mediante los cuales conviertan los datos en bruto u originales que se les presentan en una "construcción subjetiva" y significativa para sí mismos, toda vez que resulta impensable la suposición de que el conocimiento se produce por el mero traslado de información desde el lugar donde está almacenada (libros, documentos, profesores) a las cabezas-depósito de los destinatarios. Piaget identificó tal construcción con el producto de las estructuras operatorias y otros cognitivos más recientes hablan de lo mismo refieriéndose al aprendizaje significa-

Scire. 1 : 1 (en.-jun. 1995). 
tivo (Ausubel, 1976), esquemas (Norman, 1985; Glaser, 1983), guiones (Schank y Abelson, 1987), estrategias de aprendizaje (Weinstein, 1988), procesamiento primario (Sandord, 1990), etc. ¡Todo un bosque de constructos psicológicos de indudable utilidad para los documentalistas y transmisores del conocimiento e información científica!

El segundo hecho se remonta a unos pocos años atrás y me sucedió en Zaragoza. Recibí el encargo de asesorar a una Asociación de Padres de Alumnos sobre la elección de una enciclopedia para escolares del Ciclo Medio y Superior de EGB (9 a 15 años). Recorrí las más importantes librerías de la ciudad y me encontré con este dato: bajo portadas con títulos tan distintos como "Enciclopedia infantil", "Enciclopedia juvenil", "Enciclopedia universal", se ofrecían tres versiones del mismo bloque de información cuya única diferencia consistía básicamente en el número de páginas contenidas en cada una de las tres versiones. Pude observar, además, que era idéntico el vocabulario — desproporcionadamente abstracto para sus destinatarios en los tres casos-e idénticas las ilustraciones, por lo que deduje que el producto ofrecido a tan diferentes lectores estaba muy relacionado con las tijeras; las características de los destinatarios brillaban por su total ausencia.

Pasando de lo anecdótico al marco teórico de mi exposición, me propongo esclarecer qué implica actualmente, bajo el punto de vista del psicólogo, ofrecer a los consumidores del conocimiento, aprendices y lectores, los productos científicos utilizando los datos disponibles sobre el proceso del conocimiento humano, o, lo que es igual, qué modelos explican hoy el comportamiento de los sujetos a la hora de asimilar la información. Entiendo que el esclarecimiento de esta cuestión está en la base de quienes por por profesión tienen que ocuparse de los problemas de la transmisión de la información, plasmada en documentos que contengan, acoten y adapten a sus conciudadonos la casi indigerible y acelerada plétora de la producción científica. Lógicamente, dejo a los técnicos en documentación la aplicación de los datos que presento.

\section{El conocimiento humano y sus implicaciones psicológico-cogni- tivas desde los modelos de Procesamiento de Información}

Claves principales de las teorías de Procesamiento de Información. La versión psicológica de los modelos relacionados con el pensamiento humano, en general, arranca en la analogía de que el sistema cognitivo humano tendría sus correlatos (paralelismos funcionales) en el ordenador artificial, con inputs, procesos intermedios y outputs. A partir de este supuesto, la psicología cognitiva se ocupa fundamentalmente de esclarecer los procesos realizados por los sujetos humanos para codificar, almacenar y utilizar la información científica en función de cinco variables principales:

Scire. 1 : 1 (en.-jun. 1995). 
1) los tipos de conocimiento utilizados por el ser humano (Reigeluth y Stein, 1983),

2) el nivel de experiencia del sujeto dentro del campo en el que intenta asimilar nueva información (Ausubel, 1978),

3) los pasos epistemológicos o fases del pensamiento implicadas en la construcción y adquisición de los productos consolidados que llamamos ciencia, así como las leyes que relacionan el nexo entre tales fases (Flavell, 1974; Bernad, 1983),

4) la mecánica psicológica concreta o estrategias de pensamiento con las que los individuos tratan la información en el largo, original y, en gran parte hoy por hoy, inestricable camino que les conduce a la asimilación de conocimiento científico (Weinstein, 1988; Beltrán, 1987; Bernad, 1990a, 1990b). Paso a desarrollar estos cuatro apartados. Los tres últimos equivalen a lo que incluyo bajo el título de "regulación y operatividad del pensamiento humano" y, por último,

5) Influjo del factor emotivo-afectivo o ansiedad en los procesos de pensamiento.

\subsection{Tipos de conocimiento humano}

Una de las claves más importante de la psicología de Procesamiento de información es la triple clasificación del conocimiento humano en declarativo y procedimental, siendo ambos afectados, a su vez, por el metaconocimiento o conocimiento sobre el propio modo de conocer (lo que el sujeto sabe sobre lo que hace cuando piensa y sobre su propio pensamiento) (Reigeluth y Stein, 1983).

- El conocimiento declarativo o representacional -QUE SABEMOS- se ocupa de saber qué son las cosas, el mundo que nos rodea y qué hacemos para obtener una representación de la información en la mente que nos permite comprendela, almacenarla y utilizarla posteriormente. Este conocimiento está acumulado en la llamada "memoria a largo plazo" en forma de "esquemas", "redes", "mapas conceptuales", etc. que equivalen a conjuntos de datos unidos por nexos lógicos de muy distinta naturaleza y tienen su plasmación prioritaria en definiciones, leyes, fórmulas, etc.

Este tipo de conocimiento conlleva traducir los datos de la experiencia a una representación interna significativa, utilizando los dos códigos principales o básicos del pensamiento, el verbal y el gráfico (o viso-espacial).

El código verbal, asentado principalmente en el hemisferio cerebral izquierdo, procesa la información simbólica y secuencialmente (una palabra sola después de otra, un dato después de otro). Es muy abstracto y analítico y atiende a la carga semántica o conceptual de la información correspondiente. 
El código gráfico se asienta preferentemente en el hemisferio derecho, procesa la información de modo global o sintético y se ocupa de lo figurativo y estructural (relaciones globales entre los elementos, al margen de su expresión verbal o simbolización abstracta). Su característica principal es la espacialización de los conceptos implicados en los contenidos que se procesan (Williams, 1980).

- El conocimiento procedimental -COMO SABEMOS/CONOCEMOSse ocupa de cómo utilizamos la información, cómo operamos con ella y, en general, de los procedimientos que empleamos para alcanzar los conocimientos y recuperarlos cuando han de ser utilizados. Este conocimiento trabaja con la llamada "memoria operativa" - memoria a corto plazo-y utiliza dos tipos principales de procedimientos: heurísticos (directrices generales que no aseguran con eficacia el logro del objetivo pretendido, por ejemplo, aplicar un conjunto de apartados o guión para la interpretación de un texto científico); y los algoritmos (conjunto de reglas a seguir que garantizan el logro de un objetivo, por ejemplo, aplicar determinadas reglas para resolver ecuaciones lineales, realizar las operaciones aritméticas, poner en funcionamiento un aparato, etc.) (Landa, 1981).

- El conocimiento metacognitivo-QUE CONOCEMOS SOBRE NUESTRO PROPIO PROCESO DE PENSAR— estudia el conocimiento que el sujeto tiene de sí y de su sistema de pensamiento (sus recursos mentales, zonas débiles y zonas fuertes, con qué condiciones puede esperar éxito en las tareas emprendidas y qué le hace pronosticar posibles fracasos personales, cómo debe planificar sus esfuerzos para sacarles rentabilidad, etc.) (Pressley y otros, 1984; Ericsson y Simon, 1985).

Obviamente, el conocimiento de esta tipología de procesos cognitivos es una base de indudable utilidad para el documentalista.

\subsection{La regulación y operatividad del pensamiento humano}

\subsubsection{Nivel de experiencia: pensamiento del experto y del novel}

Un segundo factor que explica, en buena medida, la eficacia de los sujetos a la hora de procesar información es el nivel de experiencia adquirida dentro del campo en el que se le exige procesar nueva información. En este sentido, está comprobado que el experto realiza conductas marcadamente diferentes de las del novel. El experto es, por definición, un sujeto buen procesador de información que, sin estar especialmente dotado intelectualmente hablando, organiza con gran pericia los conocimientos dentro del campo o campos en los que posee amplia experiencia. Hoy se interpreta que tal pericia está decisivamente condicionada por los conocimientos previos (Ausubel, 1978) y esquemas mentales disponibles por el sujeto relacionados con la nueva información que procesa

Scire. 1 : 1 (en.-jun. 1995). 
(Norman, 1985). Hay que resaltar, como dato consolidado, que la pericia del experto se muestra estar circunscrita a áreas específicas por lo que no puede hablarse de expertos con carácter generalista (Pozo, 1989).

Actualmente, el interés de los psicólogos e informáticos se centra en la obtención de datos sobre el pensamiento del experto por entender que constituyen ricas directrices utilizables en el adiestramieto del pensamiento de los menos eficaces o noveles. En el cuadro siguiente, se muestran las diferencias entre la conducta del experto y del novel o principiante. (Fig. 1)

Nuestros actuales conocimientos sobre el pensamiento del experto tienen la gran ventaja que permiten a los formadores del pensamiento y transmisores de la información contar con criterios operativos manejables, aunque queda una cuestión todavía bastante confusa y por resolver para el psicólogo cognitivo: establecer las fases implicadas en el paso de novel a experto y los procesos que conlleva cada una de ellas. Esta clarificación exige todavía grandes esfuerzos de investigación básica.

2.2.2. El sistema cognitivo humano: sus posibilidades y limitaciones. El proceso que conlleva la asimilación de los conocimientos viene condicionado y modulado por la forma en que actúa el sistema cognitivo (hardware) humano y no sólo por el factor experiencia. Este sistema está sometido siempre a determinadas restricciones que, de no tomarlas en consideración, se corre el riesgo de someter a los sujetos a inútiles esfuerzos en cuanto procesadores de la información.

Datos bastante significativos y en parte consolidados, en este horizonte, son entre otros los siguientes:

- El proceso cognitivo humano arranca en los registros sensoriales del sistema nervioso central y de ellos depende básicamente. Es sabido, que los distintos registros sensoriales mantienen almacenada una representación bastante verídica de la información en el sistema central durante un período de tiempo extremadamente breve (un cuarto de segundo) (Gagné, 1991) y que sólo una pequeña fracción de esta representación completa permanece en la memoria a corto plazo durante unos diez segundos, mientras el resto desaparece del sistema; es lo que constituye la percepción selectiva..

- A lo anterior, hay que añadir que la memoria a corto plazo o memoria operativa tiene una capacidad muy limitada, $7( \pm 2)$ unidades de información (Simon, 1974). Por ello, los comunicadores de información deben contar con "este cuello de botella" a la hora de dosificar o establecer los niveles graduales de desarrollo con relación al volumen total de información que quieren transmitir (Reigeluth y Stein, 1983).

- A su vez, la información que se encuentra en la memoria operativa o a cor-

Scire. 1 : 1 (en.-jun. 1995). 
to plazo, es almacenada en la memoria a largo plazo mediante un complejo pro-

\begin{tabular}{|c|c|c|}
\hline DIMENSIONES & EXPERTO & NOVEL \\
\hline $\begin{array}{l}\text { 1.Almacenamiento de } \\
\text { información }\end{array}$ & $\begin{array}{l}\text { a) Almacena mucha } \\
\text { información } \\
\text { b) Atiende a aspectos básico y } \\
\text { esenciales de la información }\end{array}$ & $\begin{array}{l}\text { Almacena poca información } \\
\text { Atiende a aspectos } \\
\text { superficiales y concretos de } \\
\text { la información }\end{array}$ \\
\hline $\begin{array}{l}\text { 2.Codificación/ - } \\
\text { organización de la } \\
\text { información }\end{array}$ & $\begin{array}{l}\text { c) Usa códigos de representación } \\
\text { variados: verbal, icónico, } \\
\text { analógico } \\
\text { d) Organiza a partir de } \\
\text { principios y marcos generales } \\
\text { e) Clasificación/catego-rización } \\
\text { incompleta, in-consistente } \\
\text { f) Reformulación-traducción } \\
\text { personal de los datos }\end{array}$ & $\begin{array}{l}\text { Se apoya exclusivamente en } \\
\text { el código of ertado } \\
\text { Atomización del } \\
\text { conocimiento } \\
\text { Clas. completa, rigurosa, } \\
\text { consistente } \\
\text { Trabaja con los datos directos }\end{array}$ \\
\hline $\begin{array}{l}\text { 3. Control del propio } \\
\text { pensamiento } \\
\text { (metacognición) }\end{array}$ & $\begin{array}{l}\text { g) Uso amplio de heurísticos y } \\
\text { algoritmos especifícos } \\
\text { h) Amplia dedicación a la fase } \\
\text { de planificación y rapidez en la } \\
\text { de ejecución } \\
\text { i) Alta conciencia de sus zonas } \\
\text { débiles y fuertes } \\
\text { j) Variado reertorio de } \\
\text { estrategias y facilidad para } \\
\text { seleccionarlas y utilizarlas } \\
\text { k) Facilidad de transfeencia de } \\
\text { un campo a otro y tendencia a } \\
\text { la generalización } \\
\text { 1) Comprobación de resultados } \\
\text { parciales } \\
\text { ll) Calibración ajustada de } \\
\text { esfuerzos y tiempos } \\
\text { m) Síntesis de los } \\
\text { conocimientos }\end{array}$ & $\begin{array}{l}\text { Carencia de consignas } \\
\text { personales y de recursos } \\
\text { instrumentales } \\
\text { Invesrsión de tiempos con } \\
\text { respecto al experto } \\
\text { Escaso conocimiento de sus } \\
\text { propios recursos } \\
\text { No se plantea estrategias } \\
\text { alternativas } \\
\text { Individualización de los } \\
\text { datos, problemas y tareas } \\
\text { Secuencias unitarias, no } \\
\text { evalúa los objetivos parciales } \\
\text { Descontrol de tiempo y } \\
\text { esfuerzos } \\
\text { Mero listado de } \\
\text { conocimientos. }\end{array}$ \\
\hline
\end{tabular}

Figura 1. Diferencias entre el procesamiento del experto y el novel en las dimensiones congnitivas básicas

Scire. $1: 1$ (en.-jun. 1995). 
ceso de transformación o codificación, para su posterior utilización y tiene la ventaja de durar toda la vida del sujeto.

- La recuperación, por último, de la información almacenada forma parte del proceso de generación de respuestas, que implica el complicado y, por hoy, poco conocido proceso de activación-recuperación de información.

Este conjunto de datos no puede subestimarse por quienes pretenden comunicar eficamente información. Para que se entienda mejor el alcance de los mismos, basta compararlos con los homólogos realizados por el ordenador:

- La memoria operativa humana es mucho más pequeña que la unidad central de procesamiento de un ordenador ( 5 bits frente a 10 millones de bits).

- Los humanos y los ordenadores también difieren en su capacidad de almacenamiento. La memoria a largo plazo del ser humano es bastante grande (un billón de datos, que nunca, prácticamente se agota); por contra, la capacidad de almacenamiento de un ordenador es teóricamente ilimatada, solamente depende del número de cintas o de discos disponibles.

- Los tiempos de acceso a la información almacenada de los ordenadores factor decisivo en el contexto de nuestras reflexiones - son mucho menores que los del sistema humano; los primeros funcionan a la velocidad de la luz (típicamente, un microsegundo); el sistema humano, según experimentos realizados por Sternberg (1966), tarda unos 200 segundos.

- En resumen, los ordenadores poseen un espacio medio de trabajo mil veces superior al humano, pues el espacio de trabajo con que procesan la información es 20.000 veces más rápida que la nuestra y la velocidad de acceso a la información 200 veces superior a las capacidades humanas medias.

- Las anteriores desventajas y en contraste con los ordenadores, la capacidad de memorización y recuperación del ser humano depende del contexto, lo que le facilita el aprendizaje y la adaptación; en cambio, los ordenadores carecen de la capacidad de pensar soluciones nuevas, lo que constituye una clara y original desventaja con respecto a los humanos.

En la hora presente y en relación con los datos anteriores, uno de los grandes retos de la psicología cognitiva consiste en diagnosticar la capacidad real de los humanos en los diferentes contextos o escenarios en que se produce la comunicación de información. En este sentido y al margen de profundos y evidentes titubeos, se está abriendo paso, dentro del paradigma cognitivo, una metodología psicológica mediante la cual se intenta descifrar lo que se contiene en la "caja transparente" y cómo opera lo llamamos mente humana.

Una modesta muestra de estos esfuerzos es lo que un equipo de investigadores de la Universidad de Zaragoza vienen realizando desde hace casi diez años y 
que, recientemente, ha dado lugar a la publicación del proyecto "Análisis de estrategias de aprendizaje en la universidad", (1987-92) (Bernad, 1992) financiado por el M.E.C. y en el que se aportan medio millón de datos sobre la conducta de los universitarios zaragozanos en cuanto procesadores de la información. En estos momentos y con la correspondiente ayuda financiera de la D.G.A., se está llevando a cabo también el proyecto "El escolar aragonés: procesos cognitivos y aprendizajes" a través del cual pretendemos un equipo de investigadores educacionales ofrecer a los educadores de nuestra región un banco de datos primarios que puedan sustentar bases más seguras en el diseño e implementación de los proyectos instruccionales escolares.

2.3. Los pasos epistemológicos implicados en la construcción del conocimiento científico: psicología genética de Piaget

a) Objetivo de la psicología genética. Una respuesta coherente y sistemática a la cuestión de qué fases debe respetar el transmisor de conocimientos para adecuar su actuación a las posibilidades del que debe asimilarlos puede extraerse de la vasta producción científica del ya citado, Jean Piaget $(1969,1970,1978)$ y que ha quedado plasmada en su teoría genética de la inteligencia y del conocimiento (Bernad, 1983; 1990a y 1990b).

b) Estructuras operatorias y sus leyes. La psicología genética se sustancia en la afirmación de que se alcanza la ciencia en la medida en que el sujeto pone en juego, siguiendo una secuencia rigurosa e invariable, las cuatro "estructuras de pensamiento" coincidentes con las cuatro estructuras operatorias que Piaget denomina sensomotriz, preoperacional, concreta y formal y cuyos procesos más generales y significativos quedan reflejados en el cuadro siguiente (Fig. 2).

A partir del contenido cognitivo y dinámica interna de cada estructura, se

\begin{tabular}{|l|l|}
\hline ESTRUCTURAS & $\begin{array}{l}\text { PROCESOS IMPLICADOS EN CADA } \\
\text { ESTRUCTURA }\end{array}$ \\
\hline Pensamiento sensomotor & Percepciónde la realidad, de los datos de experiencia \\
\hline Pensamiento preoperacional & $\begin{array}{l}\text { Simbolización, codificación de la realidad, de losdatos } \\
\text { de exp. }\end{array}$ \\
\hline Pensamiento concreto & Análisis de la información, de los datos de experiencia \\
\hline Pesamiento formal & $\begin{array}{l}\text { Generalización de la información, de los datos de } \\
\text { experiencia }\end{array}$ \\
\hline
\end{tabular}

Figura 2. Procesos implicados en las distintas estructuras del pensamiento según PIAGET.

Scire. $1: 1$ (en.-jun. 1995). 
impondría una propuesta de procesamiento de información o macroestrategia comunicativo-asimilativa del conocimiento que, respetando la secuencialidad de las citadas estructuras, responda a los objetivos incluidos en el siguiente cuadro (Flavell, 1974) (Fig. 3):

c) Niveles de abstracción con que se presenta la información. Los datos con los que opera la inteligencia son accesibles al pensamiento en muy distinta medi-

\begin{tabular}{|c|c|c|c|}
\hline $\begin{array}{l}\text { I PENSAMIENTO } \\
\text { SENSOMOTOR }\end{array}$ & \multicolumn{2}{|c|}{$\begin{array}{c}\text { II PENSAMIIENTO } \\
\text { DEL OPERAR CONCRETO }\end{array}$} & $\begin{array}{c}\text { III PENSAMIENTO } \\
\text { FORMAL } \\
\end{array}$ \\
\hline 1 & 2 & 3 & 4 \\
\hline $\begin{array}{c}\text { Pensamiento } \\
\text { intuitivo }\end{array}$ & $\begin{array}{l}\text { Pensamiento } \\
\text { preoperacional }\end{array}$ & Operar concreto & Operar formal \\
\hline $\begin{array}{l}\text { Presencialiadad de } \\
\text { los datos } \\
\text { No procesamiento } \\
\text { lógico }\end{array}$ & $\begin{array}{l}\text { Egocentrismo } \\
\text { Centraje } \\
\text { Transducción } \\
\text { Irreversibilidad }\end{array}$ & $\begin{array}{c}\text { Composición } \\
\text { Asociatividad } \\
\text { Identidad } \\
\text { Reversibilidad }\end{array}$ & $\begin{array}{l}\text { Pens. hipot.-ductivo } \\
\text { Pens. proposicional } \\
\text { Pens. combinatorio }\end{array}$ \\
\hline
\end{tabular}

Figura 3. Estructuras de pensamiento, procesos que implican.

da en función de su nivel de abstracción o, lo que es igual, del modo de hacerse presentes en el pensamiento. No se facilita en la misma medida la comprensión de un fenómeno químico, por ejemplo, pidiendo al sujeto que imagine lo que sucederá si se hace tal o cual experimento que mostrándoselo en el laboratorio directamente; ni es igualmente comprensible la novela social de nuestro siglo a partir de resúmenes contenidos en libros de texto que a partir de la lectura de algunos autores típicamente representativos. Los ejemplos se podrían multiplicar. Este presupuesto es decisivo para quienes se ocupan de transmitir información, pues es obvio que, en contra de lo que pudiera sugerir el sentido común, la información científica que se sumistra a unos determinados usuarios está constituida por productos altamente elaborados que responden a concepciones fruto de un sofisticado proceso de abstracción.

En el cuadro siguiente se reflejan los grados de abstracción con que puede ofrecerse una información, en general, ejemplos de cada uno de ellos, así como la enumeración de los procesos prototípicos que comporta cada nivel de abstracción (Fig. 4): 
Como ilustración concreta de los diversos grados de abstracción, describamos un caso de utilidad para quienes se ocupan de elaborar fuentes de información para hipotéticos lectores o aprendices. En el campo de las Ciencias podrían pensarse los siguientes grados de abstracción: al lector de un documento se le invita a que vaya y observe una reacción química en el laboratorio (Grado 0), active el recuerdo de las propiedades específicas de un gas determinado que estudió anteriormente (Grado 1), deduzca la naturaleza del combustible que arde en una caldera a partir de las propiedades de un grupo determinado de gases o calcule el número de moléculas de aire contenidas en una habitación de unas concretas dimensiones y con una temperatura de $\mathrm{X}$ grados y $\mathrm{Z}$ mm de presión (Grado 2).

\subsection{Tipología de estrategias de pensamiento en contextos disciplinares y} transmisión de información

\subsubsection{Aclaraciones epistemológico-conceptuales}

A la hora de enfrentarse con la elaboración de una escala relativa a la Tipología de estrategias de pensamiento de utilidad para los documentalistas, hay que ponerse de acuerdo en el punto de partida, qué se quiere decir con la expresión "estrategia de pensamiento". Esto es tanto más necesario cuanto que, hoy por hoy, es patente la falta de claridad sobre este complejo constructo, que es usado, con bastante alegría y sin tener en cuenta la variopinta polisemia que lo caracteriza. Por otra parte, entendemos que uno de los más valiosos instrumentos para los transmisores de información pasa por entender cómo puede y suele asimilarla el sujeto humano.

A partir de las anteriores premisas, es necesario precisar que en nuestro planteamiento, entendemos por "estrategia de pensamiento" el conjunto de procesos que el sujeto realiza con vistas a la consecución de un objetivo: asimilar información nueva. En el encabezamiento de esta parte, hemos añadido "en contextos disciciplinares" por entender que es difícil sustentar la validez de cualquier tipología del pensamiento humano con carácter general y directamente aplicable en todas las áreas del conocimiento. La experiencia enseña y los datos científicos lo ratifican que sólo muy limitadamente los sujetos transfieren sus conductas cognitivas de unos campos a otros.

Añadamos que, para nuestro propósito, las notas más decisivas del concepto de "estrategia" son tres: 1) la conciencia, por parte del sujeto, de la existencia de una meta relativa a la tarea que ejecuta, 2) la conciencia relacionada con la planificación de las acciones encaminadas a la consecución de dicha meta, y 3 ) el control-evaluación de la ejecución previamente planificada.

El conocimiento que el sujeto tiene de los tres pasos mencionados constituye

Scire. 1 : 1 (en.-jun. 1995). 
lo que se llama "metacognición” y que, por su propia naturaleza, afecta en mayor o menor grado a los tres componentes de referencia. En síntesis, pues, nuestro concepto de estrategia de pensamiento equivale a un conjunto organizado y consciente de un proceso de información complejo y conducente a una meta.

2.4.2. Tipología de estrategias de pensamiento, variables y niveles de elaboración de la información por los sujetos humanos

La tipología y escala que siguen ofrecen un análisis pormenorizado de cada una de las estrategias que integran un procesamiento de información global típicamente humano. En la elaboración de esta escala distinguimos: 6 dimensiones, 10 grandes estrategias y, dentro de éstas, 20 variables (VAR), con tres niveles (N) por variable.

Huelga el recordar que, para el psicólogo cognitivo, la meta última de esta elaboración es conseguir un diagnóstico válido, en términos de "perfiles" o tipos, de la conducta de los humanos en cuanto procesadores de información; entendiendo que el establecimiento de tales perfiles permitirán diseñar, de manera más eficaz, las estrategias de transmisión de información, objetivo final de los profesionales aquí reunidos.

Al llegar a este punto, se hace necesaria una observación: la adecuada aplicación de esta tipología de procesos de pensamiento requiere siempre que sea "traducida" o acomodada a las exigencias concretas impuestas por la naturaleza y nivel de los procesos de información implicados en las correspondientes tareas que interesa comprender.

\section{DIMENSION: DOMINIO GENERAL DEL TEMA}

Esta Dimensión es una especie de síntesis del conjunto de procesos resultante del enfoque general seguido por el sujeto en su procesamiento de información, y dice relación con los procesos que describen la forma de realizar la comprensión del núcleo temático que se procesa o tarea que se realiza: estructural/sintético y secuencial/analítico.

Nos lleva a pensar en cierto paralelismo entre los modos de asimilar la información procesada y el doble código de representación del pensamiento humano: síntesis (hemisferio derecho o representación global) y análisis (hemisferio izquierdo o representación verbal y secuencial).

Hipotetizamos, además, que las estrategias implicadas en la asimilación de nuevos contenidos son dependientes, principalmente, de los conocimientos previos o esquemas mentales del sujeto.

En cualquier caso, lo que medimos aquí es la habilidad del sujeto para desglosar cognitivamente los temas concretos con que se enfrenta en función de dos

Scire. $1: 1$ (en.-jun. 1995). 
trayectorias posibles diferentes, yendo desde la globalidad al procesamiento analítico de los datos o, al contrario, desde su visión analítica a la sintética. En uno y otro caso, hay que tener en cuenta el conjunto de vicisitudes que pueden darse dentro de cada una de las trayectorias o enfoques (errores, lagunas, sesgos, etc.).

\section{$1^{a}$ Estrategia: Estructura o secuenciación global del proceso}

Hace referencia a dos enfoques o estrategias generales claramente distintas seguidas por el sujeto a la ahora de enfrentarse con la actividad peculiar implicada en una tarea que requiera procesar información, lo que se traduce en un tipo de procesamiento de la nueva información a partir de una estrategia preferentemente analítica o sintética.

Lógicamente, para interpretar la actividad del sujeto, se le debe facilitar la toma de contacto inicial con el contenido de la tarea e invitarle a que diga qué va a hacer para llevarla a cabo.

- $1^{a}$ Variable: Síntesis o globalidad. Desde esta estrategia, el sujeto intenta captar o comprender los contenidos teniendo en cuenta la idea general y estructura básica de los mismos, lo que implica un proceso de globalización o de síntesis del tema/conceptos en cuestión, sin "perderse" o quedarse en los datos concretos y particulares. La escala de valores de la variable es la siguiente:

I Nivel: $\quad$ El sujeto hace referencia al tema u objeto material de la tarea en sí misma. Ejs. "Es un ejercicio de quebrados y de números enteros", "se habla del SIDA", "se trata de un texto y de unas cuantas preguntas sobre el mismo", etc.

II Nivel: El sujeto hace referencia al contexto conceptual preciso en el que queda enmarcado el tema y la tarea que implica dominarlo. Ejs. "Es un texto que tengo que leer e interpretar", "se me exige resolver problemas a base de operaciones aritméticas", "debo analizar o resumir el texto, hablar de sus personajes", etc.

III Nivel: Añade a lo anterior la síntesis en expresión personal y propia del tema aludiendo a la naturaleza de la tarea, en general, que está en juego y sin apenas citar ideas concretas contenidas en el original propuesto; o, también, alude a un conjunto de procesos o tratamientos que han de realizarse a partir de los ejercicios propuestos. Ejs. " Con este texto puedo hacer un resumen, analizarlo, hacer un esquema...", "tengo que pronunciarme sobre los problemas que plantea el SIDA, tras haber tenido en cuenta todo lo que sé sobre él y lo que aquí se dice", etc.

- $2^{a}$ Variable: Análisis. En este caso, a diferencia del anterior, el sujeto centra su atención en las partes del contenido sin ocuparse o formarse idea del todo, 
se "siente perdido" o "atrapado" por las partes del tema y se queda en ellas. La escala de valores de la variable es la siguiente:

I Nivel: El sujto se limita a mencionar alguna o algunas de las partes o ideas particulares propuestas.

II Nivel: Asigna o menciona algunos de los tipos de actividad distintos que se le exigen realizar o algunas partes de la tarea con las implicaciones correspondientes.

III Nivel:Enumera con clara sistematicidad los principales ideas que se le ofrecen y los procesos prototípicos que se le exigen para asimilarlas.

\section{$2^{\circ}$ Estrategia: Aciertos típicos}

$\mathrm{El}$ acierto puede referirse a las siguientes variables:

- $3^{a}$ Variable: Planteamiento. Esta estrategia se ocupa de la demarcación del tema y comprende los procesos que conlleva el situarlo en un contexto preciso y apropiado. La escala de valores de la variable es la siguiente:

I Nivel: Los plantemientos del sujeto son en su mayoría falsos y acierta sólo alguno, o, también, salta sin lógica de un planteamiento a otro, cayendo en contradicciones.

II Nivel: Plantea adecuadamente la mitad aproximada de las cuestiones o aspectos implicados en la tarea, los enfoca correctamente.

III Nivel: Plantea correcta y lógicamente prácticamente todas las cuestiones.

- $4^{a}$ Variable: Partes acertadas por el sujeto. Analiza los procesos que conducen a determinar/comprender las partes del tema o tarea sin cometer errores o sesgos y, por tanto, sin necesidad de rectificar - "acierta a la primera". La escala de valores de la variable es la siguiente:

I Nivel: El sujeto demuestra no comprender apenas el fondo de la tarea, se manifiesta como perdido con relación a ella y sus implicaciones.

II Nivel: El sujeto acierta alrededor de la mitad de las cuestiones implicadas en la tarea, tanto en su planteamiento como en su desarrollo.

III Nivel: El sujeto entiende el objeto o metas de la tarea y plantea y ejecuta correctamente sus distintas partes.

Una consigna general para obtener los datos que interesan a la I y II dimensión puede ser ésta:

"Lea primero todas las CUESTIONES que se le presentan y tras la lectura atenta de las mismas, diga de qué tratan, a qué se refieren y qué puede y deberá hacer con respecto a tales CUESTIONES". 
(Recuérdese que las consignas han de ser abiertas para que el sujeto tenga ocasión de orientar su propia interpretación de la tarea en un sentido u otro).

II DIMENSION: ERRORES, LAGUNAS Y DUDAS TIPICAS

Esta II Dimensión viene a ser el negativo (falta de dominio del tema) analizado en la I Dimensión, y equivale a la explicitación de la tarea en sus componentes analíticos y sintéticos ya considerados en la I Dimensión, aunque ahora en sentido negativo. La II Dimensión engloba las siguientes estregegias:

$3^{a}$ Estrategia: errores típicos (que pueden derivarse de):

- $5^{a}$ Variable: presupuestos falsos.

- $6^{a}$ Variable: datos falsos.

- $7^{a}$ Variable: falsa generalización.

- $8^{a}$ Variable: falsas deducción/inducción.

- $9^{a}$ Variable: lapsus.

La escala de medida para este grupo de variables es la siguiente:

I Nivel: El sujeto comete muchos errores en la tarea que realiza, implicados en las Variables $5^{\mathrm{a}}$ a la $9^{\mathrm{a}}$.

II Nivel: Comete solamente algunos errores importantes, derivados principalmente de alguna o algunas de las Variables $5^{\mathrm{a}}$ a la $8^{\mathrm{a}}$.

III Nivel: No comete prácticamente ningún error de importancia o algún lapsus en la ejecución de la tarea.

$4^{a}$ Estrategia: lagunas típicas

- $10^{a}$ Variable: omisiones esenciales relativas al contenido que procesa o tarea.

- $11^{a}$ Variable: omisiones secundarias del tema. La escala es la siguiente:

I Nivel: El sujeto demuestra lagunas importantes tanto en el planteamiento como ejecución de la tarea.

II Nivel: Tiene alguna laguna importante y otras secundarias en la tarea realizada.

III Nivel: No aparecen lagunas significativas en el enfoque o desarrollo de la tarea.

$5^{a}$ Estrategia: dudas típicas

- $12^{a}$ Variable: en el planteamiento inicial y subsiguientes.

Scire. $1: 1$ (en.-jun. 1995). 
-13 Variable: en la ejecución.

La escala es la siguiente:

I Nivel: El sujeto muestra muchas dudas o algunas muy significativas tanto en el planteamiento como en el desarrollo de la tarea.

II Nivel: Aparecen algunas dudas de relativa importancia en el planteamiento o desarrollo de la tarea.

III Nivel: Apenas aparecen dudas en el planteamiento o desarrollo de la tarea.

$6^{a}$ Estrategia: Hipótesis utilizadas y su permanencia/cambio

- $14^{a}$ Variable: cambio/utilización de distintos enfoques. La escala es la siguiente:

I Nivel: El sujeto muestra variedad de indecisiones en el planteamiento, desarrollo o ejecución de la tarea o, también, utiliza planteamientos contradictorios.

II Nivel: Muestra algunas indecisiones, inconsistencias en el planteamiento o ejecución de la tarea.

III Nivel: Apenas muestra indecisiones en el planteamiento o ejecución de la tarea.

III DIMENSION: DOMINIO DE LENGUAJES O CODIGOS DE REPRESENTACION

Esta III Dimensión analiza la calidad o dominio del sujeto de los diferentes códigos de representación del conocimiento y especialmente el vocabulario o código verbal.

$7^{a}$ Estrategia: uso de lenguajes: verbal, icónico y analógico

- $15^{a}$ Variable: lenguaje verbal (precisión y suficiencia). La escala es la siguiente:

I Nivel: El sujeto demuestra un dominio escaso del lenguaje verbal.

II Nivel: Demuesta cierto dominio del lenguaje verbal.

III Nivel: Demuestra gran dominio (precisión y riqueza) en el lenguaje verbal.

Consigna: "Haga un resumen del texto que acaba de leer o tema que se le plantea procurando decirlo en palabras distintas de las que aparecen en él (Lengua)"."'Cuente con sus propias palabras alguno de los problemas que ha resuelto (Matemáticas)". 
- $16^{a}$ Variable: lenguaje icónico. La escala es la siguiente:

I Nivel: El sujeto demuestra muy escasas posibilidades de representarse los conocimientos mediante el lenguaje icónico.

II Nivel: Demuestra ciertas posibilidades en el campo de la representación icónica del conocimiento.

III Nivel: Se muestra experto en el dominio del lenguaje icónico.

Consigna: "Haga varios dibujos que representen lo mejor posible las ideas importantes del texto (Lengua), "Haga un dibujo de los problemas que ha resuelto (Matemáticas)".

- $17^{a}$ Variable: lenguaje analógico. La escala es la siguiente:

I Nivel: El sujeto apenas sabe reconocer, utilizar o crear analogías.

II Nivel: Es capaz de utilizar y crear algunas analogías.

III Nivel: Utiliza con facilidad analogías.

Consigna: "Analice la metáfora X. Invente, por su cuenta otra”. “Es una metáfora $X$ - se propone una-. Diga por qué sí o por qué no (Lengua)". "Formule otros problemas semejantes a éstos (Matemáticas)".

IV DIMENSION: CALIDAD DEL RAZONAMIENTO LOGICO O PROCESOS INFERENCIALES

Esta IV Dimensión estudia el rigor de la lógica utilizada por el sujeto.

$8^{a}$ Estrategia: momentos diferenciales y calidad del razonamiento lógico

Estudia la precisión de todo tipo de inferencias realizadas por el sujeto, deducción/inducción, dentro de la resolución de tareas en que procesa información.

- $18^{a}$ Variable: inferencia. La escala es la siguiente:

I Nivel: El sujeto apenas tiene en cuenta el paso de antecedentes a consecuentes: paso de los principios o definiciones a sus aplicaciones, de lo que dice con lo que ha dicho, o cambia, sin lógica, el método de unos ejercicios a otros incoherentemente, etc.

II Nivel: Utiliza una lógica en parte equivocada o incoherente a lo largo de la tarea.

III Nivel: Muestra un discurso lógicamente coherente a lo largo de la tarea.

V DIMENSION: GRADO DE ABSTRACCION

Esta V Dimensión indica el nivel de abstracción con que el sujeto domina la

Scire. $1: 1$ (en.-jun. 1995). 
información que procesa. Se entiende por "abstracción" la distancia entre los datos inmediatos que se le proporcionan y la traducción y generalización que hace de los mismos.

$9^{a}$ Estrategia: Grado de abstracción con que se dominan los contenidos temáti$\cos$

- 19 Variable: abstracción. La escala es la siguiente:

I Nivel: El sujeto informa de modo nada sistemático de los contenidos de la tarea o se limita básicamente a enumerar unos cuantos datos o contenidos de la misma; se comporta como un mal "repetidor" de lo que tiene delante.

II Nivel: Añade alguna mención a datos que no se contienen materialmente en las tareas que le son propuestas y, sin embargo, dicen relación con ella; introduce, por su cuenta, alguna separación o estructuración de los contenidos de la tarea, etc.

III Nivel: Añade a lo anterior algún tipo de estructuración "inclusiva" —es decir, completa-, o de generalización del conjunto de apartados incluidos en la tarea, por ejemplo, relacionando sus contenidos con otros temas del área o disciplina afín (transfer).

\section{DIMENSION: CONCIENCIA COGNITIVA}

Esta VI Dimensión (metaconocimiento) se refiere a la conciencia personal del sujeto - lo que piensa y sabe decir- sobre los componentes personales implicados en la planificación y ejecución de las tareas que emprende o ha realizado.

Consta, entre otros, de tres componentes principales: 1) la conciencia que tiene con relación a sentirse seguro de que entiende lo que se le pregunta o la respuesta que se le exige (qué hace para definir sus objetivos o metas), 2) igualmente, la conciencia puesta en juego en el proceso de autoevaluarse tanto sobre el grado en que va consiguiendo los subobjetivos, paso a paso, como sobre la adecuación de los procedimientos que utiliza para conseguirlos, y 3) su nivel de conciencia con relación al mantenimiento o modificación "sobre la marcha" de las estrategias utilizadas, lo que tiene que hacer para superar las dificultades que experimenta, dar por terminado un proceso o seguir en el empeño, etc.

Es sabido que los sujetos expertos conocen mejor que los noveles sus zonas débiles, calibran más y mejor los diferentes pasos que siguen para organizar/asimilar la información que utilizan y aprovechan su experiencia de éxito o fracaso en las tareas que emprenden. 
$10^{a}$ Estrategia: metacognición

- $20^{a}$ Variable: metaconocimiento o conocimiento que tiene el sujeto sobre su propio proceso de pensar. La escala es la siguiente:

I Nivel: El sujeto no menciona para nada lo que hace para planificar o ejecutar su actividad y se limita a mencionar el contenido material de la tarea que se le propone.

II Nivel: Alude a algunos de los procesos (fases, facilidad o dificultad, dudas, errores, etc.) implicados en la resolución de la tarea.

III Nivel: Hace clara referencia a las propias dificultades, dudas, errores, temores, etc. inherentes a su modo de organizar y ejecutar la actividad desarrollada para alcanzar la meta que se propone. Este tipo de sujeto actúa con la conciencia de que su actividad mental es algo propio y responsable de ella.

Consigna: COMO PIENSA (Se presentará al sujeto un folio al final de la tarea y tras haberla terminado, con la consigna que sigue. Estos mismos datos se podrán obtener a través de una entrevista)

"Ha termindo de contestar lo mejor que ha podido a las CUESTIONES que se le han presentado. Ahora se trata de que diga:

1) Todo lo que cree que ha hecho para contestarlas: diga con sus propias palabras y lo más claramente que sepa el modo como $\mathrm{Vd}$. ha ido pensado a lo largo de sus contestaciones y lo que ha hecho para realizarlas bien.

Seguidamente, y suponiendo que tuviera que explicar a un amigo todo lo referente a lo que ha hecho durante el tiempo dedicado a responder a las CUESTIONES propuestas, diga:

2) Tres momentos o puntos en los que se ha sentido seguro de que lo que iba pensando era lo correcto.

3) Tres dudas que le han surgido a lo largo de la tarea que ha terminado y qué ha hecho para resolver bien, aunque no lo haya conseguido del todo, y

4) Tres errores que Vd. mismo se ha corregido, es decir, después de dar una solución falsa o utilizar un método inadecudo, ha pensado en otra solución mejor o ha cambiado el método.

\section{Influjos del factor emotivo-afectivo o ansiedad en los procesos de pensamiento}

Además del conjunto de procesos, de naturaleza básicamente mental, analizado por medio de la anterior tipología, es de interés, a veces decisivo, el sopesar en qué medida la ansiedad obstaculiza o facilita el procesamiento de información

Scire. 1 : 1 (en.-jun. 1995). 
por parte de los sujetos. Un vicio frecuente entre los analistas del pensamiento es prescindir de los influjos de lo emocional en el discurso mental de los individuos.

Para calibrar este factor de ansiedad, pueden aplicarse instrumentos psicotécnicos existentes en el mercado pero hay que reconocer que su estandarización excesiva generalidad de la medición - hace que no sean una apreciación válida del factor ansiedad vivida por los sujetos humanos en cada contexto concreto (Sternberg, 1985). Para subsanar las limitaciones de estos instrumentos, se propone aquí una consigna múltiple destinada a medir este factor en el interior de la tarea.

CONSIGNA (Encierra en un circulito las respuestas que elige):

- Al principio:

1) "Para resolver las CUESTIONES que tiene delante, piensa que: a) Está mal preparado y casi seguro de que le va a salir mal la tarea que va a realizar. b) Poco preparado y le saldrá probablemente regular o a medias el trabajo que va a realizar. c) Bastante preparado y probablemente le saldrá bastante bien el trabajo. d) Muy bien preparado y le saldrá muy bien todo o casi todo loque implica hacer bien la tarea con que se enfrenta.

- Hacia la mitad:

2) El que le puedan salir estos ejercicios peor que a otros compañeros o amigos, le preocupa: a) Mucho. b) Bastante. c) Poco d) Nada o casi nada

3) Desde que ha comenzado a contestar las preguntas que se le han formulado, ¿cuántas veces ha pensado que le puede faltar tiempo o va a fallar a la hora de contestarlas bien: a) Muchas veces b) Bastantes veces c) Pocas veces c) Ninguna vez

- Al final:

4) Le preocupa que haya podido fallar en este trabajo: a) Mucho. b) Bastante. c) Poco. d) Nada o casi nada

5) Terminada este trabajo, teniendo en cuenta cómo lo ha hecho y siendo totalmente sincero consigo mismo, piensa que es: a) Una persona muy eficaz que casi siempre hace bien las tareas que le proponen. b) Una persona que bastantes veces acierta en lo que hace. c) Un persona que acierta pocas veces en las tareas y trabajos que hace aunque de vez en cuando acierta. d) Un persona que rara vez o casi nunca acierta a la hora de hacer las tareas.

\section{Campos abiertos a la investigación en la psicología del pensamiento}

Los planteamientos anteriores son una muestra no sólo de la complejidad del tema aquí tratado sino, y sobre todo, de los retos que tiene delante la psicología Scire. $1: 1$ (en.-jun. 1995). 
cognitiva. En ese sentido, un listado básico de tareas que esperan clarificación coinciden con las "asignaturas pendientes" de quienes ahora trabajamos en el diagnóstico del pensamiento y aprendizaje humanos:

1) Márgenes de modificabilidad estructural de los sujetos en su actividad cognitiva. Un postulado sostenido por los más optimistas (Fuerstein, 1982) cree que es posible afirmar la adaptabilidad de las personas hasta el límite de conseguir una reestructuración de su dinámica mental. Evidencias parciales lo confirmarían.

2) Nivel de participación de cada hemisferio en el procesamiento de información, así como la corresponsabilidad bihemisférica conjunta en tal procesamiento.

3) Las etapas evolutivas de los sujetos en cuanto a su adiestramiento como procesadores de información, así como la transferibilidad de procesos mentales de unos campos a otros del saber. Este punto se considera decisivo en los ambientes académicos, fuertemente jerarquizados cognitivamente hablando.

4) Nexos cognitivos de dependencia e interacción entre la naturaleza esspecífica de los contenidos y datos que se procesan y los procesos peculiares necesarios para su procesamiento (Stodolsky, 1991).

Prácticamente, todos estos capítulos están en relación con el primero citado y vienen a ser un desglose del mismo.

\section{Apendice: Cinco cuestiones principales sobre el conocimiento humano según las teoría cognitivas y procesamiento de la información}

$\left.1^{a}\right)$ Tipos de conocimiento humano:

a) Declarativo o representacional (Qué conocemos)

— Esquemas, redes semánticas, mapas conceptuales

-2 códigos de representación:

- Verbal (hemisferio izquierdo)

- Gráfico (hemisferio derecho)

b) Procedimental (Cómo conocemos)

- Algoritmos

- Heurísticos

- Sistemas "computacionales" o de naturaleza idiosincrática /diferencial ("Estrategias de aprendizaje" de cada sujeto)

c) Metaconocimiento (Que sabemos de nuestro proceso de conocer)

Scire. $1: 1$ (en.-jun. 1995). 
- Utilización de la experiencia de aprendizaje/conocimiento

- Planificación y control del proceso de pensar

$\left.2^{a}\right)$ Nivel de experiencia y regulación del pensamiento humano

a) Pensamiento del experto y del novel: una clave útil pero insufiente

b) Sistema cognitivo humano: el software básico humano

$\left.3^{a}\right)$ Los pasos fundamentales del proceso constructivo de la ciencia: la epistemología genética de J. Piaget

a) Objetivo de la psicología genética: proceso creciente de "adaptación"

b) Estructuras operatorias y sus metas

c) Niveles de abstracción en el tratamiento de la información

$\left.4^{a}\right)$ Estrategias de pensamiento: el "software secundario" del conocimiento humano: estrategias de apendizaje

a) La "estrategia de pensamiento": unidad computacional básica de la cognición

b) Un modelo dimensionado del proceso de pensar/aprender

c) Variables del proceso de pensar

$\left.5^{a}\right)$ Factor emocional en el entorno del conocimiento: "Efecto pygmalion"

\section{Referencias}

Alexander, P. A. y Judy, J. E.(1988). The Interaction of Domain Specific and Strategic Knowledge in Academic Performance. // Review of Educational Reseach. 4 (1988) 375-404.

Ausubel, D. P. (1978). Educational Psychology : a cognitive View. 2th ed. New York: Holt, 1978.

Beltrán, J. (1987). Estrategias de aprendizaje. // Beltrán (ed.). Psicología de la educación. Madrid : Eudema, 1987. 118-139.

Bernard, J. A.(1983). Factores y procesos de aprendizaje : Una didáctica basada en J. Piaget. Zaragoza : Universidad, ICE, 1983.

Bernard, J. A.(1990a). Psicología de la enseñanza-aprenidzaje en el Bachillerato y Enseñanza Profesional. Zaragoza : ICE-Universidad, 1990.

Bernard, J. A.(1990b). Estrategias de enseñanza-aprendizaje en la universidad. Zaragoza: ICE-Universidad, 1990.

Bernard, J. A. (dir.) (1992). Analisis de las estrategias de aprendizaje en la universidad : Informe final del Proyecto : 1987-92. Zaragoza : Universidad, ICE, 1992.

Ericsson, K. A. ; Simon, H. A. (1985). Protocol Analysis : Verbal Reports as Data. London : The MIT Press, 1985. 68-79.

Feuerstein, R. (1982). Instrumental Enrichment : An Intervention Program for Cognitive

Scire. 1 : 1 (en.-jun. 1995). 
Modificability. Baltimore : University Park Press, 1982.

Flavell, J. A. (1974). La psicología de J. Piaget. Buenos Aires : Paidós, 1974.

Gagne, H. (1991). La psicología cognitiva del aprendizaje escolar. Madrid : AprendizajeVISOR, 1991.

Giordan, A. ; Vecchi, G. de (1988). Los orígenes del saber : De las concepciones personales a los conceptos científicos. Sevilla : Diada, 1988.

Glaser, R. (Ed.) (1983). Advances in Instructional Psycholgy. New Jersey : L. E. A., 1983. Vol. 1 y 2 .

Landa, L. N. (1981). La capacidad de pensar: ¿Cómo puede enseñarse?. // Pérez y Almaraz: Lecturas de aprendizaje y enseñanza. Madrid : Zero, 1981.

Monereo, C. (comp.) (1990). Enseñar a aprender y a pensar enl a escuela. // Ponencias de las I Jornadas de estudio sobre estrategias de aprendizaje. Madrid : Infancia y Aprendizaje, 1990.

Nickerson, R. S. ; et al. (1985). The Teaching of Thinking. New Jersey: L. E. A., 1985.

Norman, D. A. (1985). El aprendizaje y la memoria. Madrid : Alianza, 1985.

Piaget, J. (1969). Biología y conocimiento. Madrid : Alianza, 1969.

Piaget, J. (1970). Psicología de la inteligencia. Buenos Aires : Psique, 1970. Pub. original en 1947.

Piaget, J. (1978). La equilibración de las estructurs cognitivas : Problema central del desarrollo. Madrid : Siglo XXI.

Pozo, J. I. (1989). Teorías cognitivas del aprendizaje. Madrid : Morata, 1989.

Pressley, M. ; et al. (1984). Metamorystrategy monitoring in adults and children. // Journal of Verbal Learning and Verbal Behavior. 23 (1984) 270-288.

Reigeluth, C. H. ; Stein, F. S. (1983). The elaboration theory of instruction. // Reigeluth (ed.). Instructional Desing : Theories and Models. Hillsdale ; New Jersey : Erlbaum, 1983. 335-381.

Sandord, A. (1990). La mente del hombre. MAdrid : Alianza, 1990.

Schank, R.C. ; Abelson, R. P. (1987). Guiones, planes, metas y entendimiento. Barcelona : Paidós, 1987.

Simon, H.A.(1974). How big is a chunk? // Science. 183 (1974) 482-488.

Sternberg, J. R. ; Powell, J. S. (1985). Handbook of intelligence. London : Cambridge Univ. Press.

Stodolky, S. S. (1991). La importancia del contenidos en la enseñanza. Barcelona : Paidós-MEC, 1991.

Weinstein, C. E. (1988). Assessment and Training of Student Learning Strategies. // Schmeck (ed.). Learning Strategies and Learning Styles. London : Plenum Press, 1988. 291-316. 